\title{
DETERMINAÇÃO DE HERBICIDAS USADOS NO CULTIVO DE ARROZ IRRIGADO NA REGIÃO SUL DO ESTADO DE SANTA CATARINA ATRAVÉS DA SPME-GC-ECD
}

Léa L. F. Costa e Ernani S. Sant'ana

Departamento de Ciências dos Alimentos, Universidade Federal de Santa Catarina, CP 476, 88040-900 Florianópolis - SC, Brasil Eliane A. Suchara, Vanira S. Benato e Eduardo Carasek*

Departamento de Química, Universidade Federal de Santa Catarina, CP 476, 88040-900 Florianópolis - SC, Brasil

Recebido em 21/12/06; aceito em 21/6/07; publicado na web em 19/12/07

\begin{abstract}
DETERMINATION OF HERBICIDES USED IN IRRIGATED RICE CULTIVATION IN THE SOUTH OF SANTA CATARINA USING SPME-GC-ECD. Evaluation of the pollution by the herbicides alachlor, propanil and atrazine in water samples from four rivers in the cities of Turvo and Meleiro, south of Santa Catarina State, was made using the SPME-GC-ECD method. The proposed method was optimized and validated. The correlation coefficients were higher than 0.997 and linear ranges of the analytical curves were 0.1-4; 0.1-2.5 and 0.1-5 $\mu \mathrm{g} \mathrm{L}^{-1}$ for atrazine, alachlor and propanil, respectively. The herbicides were quantified by GC-ECD and identified by GC-MS. Both of the selected rivers presented contamination by at least one of the studied herbicides.
\end{abstract}

Keywords: herbicides; river water; SPME.

\section{INTRODUÇÃO}

A região sul catarinense é abastecida pela bacia hidrográfica do rio Araranguá, sendo seus principais afluentes os rios Manoel Alves, Amola-Faca e Itoupava. Estes rios têm uma vital importância para região sul de Santa Catarina, pois eles banham as áreas agriculturáveis localizadas as suas margens. Eles beneficiam os rizicultores de todo vale e contribuem também para outras culturas, pecuária, pesca, turismo, bem como o abastecimento de água potável para os habitantes da região.

Em Santa Catarina, o cultivo de arroz é realizado $100 \%$ no sistema pré-germinado, alcançando uma produtividade ao redor de 7.000 $\mathrm{kg} \mathrm{ha}^{-1}$, em uma área de 126 mil ha. O Estado ocupa o segundo lugar na produção de arroz irrigado, com cerca de 800 mil t anuais, sendo o sul do estado responsável por $42 \%$ do total da produção ${ }^{1}$.

No entanto, existe a preocupação em relação ao uso indiscriminado dos pesticidas que após aplicação são absorvidos pelas argilas contidas no solo e permanecem expostos às inúmeras reações químicas, físicas e biológicas. Desta forma, sabe-se que a aplicação destes produtos é perceptível pelo odor característico que se constata em cidade como Turvo e Meleiro ${ }^{1}$. Geralmente, a população costuma atribuir os elevados níveis de turbidez dos rios, principalmente na época do preparo de canchas de arroz, à contaminação por pesticidas utilizados na lavoura de arroz.

O uso generalizado e intensivo de pesticidas tem gerado diversos problemas relacionados à saúde pública e ao desequilíbrio ambiental, tais como intoxicações de agricultores, contaminações de alimentos, água e solos, resistência de espécies combatidas às substâncias empregadas e danos em espécies não visadas ${ }^{2}$. O desequilíbrio ecológico ocasionado acarreta problemas à própria agricultura, agravando o surgimento e proliferação de pragas e doenças e, conseqüentemente, aumento da necessidade de usar mais pesticidas ${ }^{3}$.

O despejo de substâncias tóxicas utilizadas na agricultura é uma das fontes de contaminação das águas, pois cada vez mais utilizamse inseticidas, herbicidas, fungicidas e toda uma série de praguicidas.

*e-mail: carasek@qmc.ufsc.br
Mesmo que estas substâncias cheguem aos rios e lagos em pequenas quantidades, a bioacumulação fará com que sua ação seja prejudicial ao longo da cadeia alimentar, que inclui os seres humanos.

Órgãos internacionais como a "Environmental Protection Agency" (EPA) dos Estados Unidos e da Comunidade Européia (EC) iniciaram um controle estabelecendo limites em relação às concentrações de pesticidas encontrados em água. Os níveis permitidos pela EC são determinados pela "Drinking Water Directive" (80/778/EEC $)^{4}$ que estabeleceu que a concentração máxima tolerável de um pesticida individual não deve exceder $0,1 \mu \mathrm{g} \mathrm{L}^{-1}$ e que a concentração total de pesticidas não deve exceder $0,5 \mu \mathrm{g} \mathrm{L}^{-1} \mathrm{em}$ água potável. O Brasil apresenta uma legislação que também regulamenta os níveis máximos de pesticidas em água potável, baseados em sua periculosidade. A Portaria do Ministério da Saúde $n^{\circ}$ 518, de 25 de março de 2004, estabelece os padrões de potabilidade para substâncias químicas que representam risco à saúde 5 .

Os herbicidas alaclor (2-cloro-N-(2'6'-dietilfenil)-N(metoximetil) acetamida), atrazina (2-cloro-4-etilenodiamino-6isopropilamino-s-triazina) e propanil (3-metil-3,4-diclo-roacetanilida) são amplamente aplicados nas culturas de algodão, soja, amendoim, café, cana-de-açúcar, milho e arroz. São considerados produtos de mediana a extremamente tóxicos, sendo muito perigosos para o ambiente, tendo seus limites máximos para água de consumo humano estabelecido pela legislação brasileira em $2 \mu \mathrm{g} \mathrm{L}^{-1}$ para atrazina e $20 \mu \mathrm{g} \mathrm{L}^{-1}$ para alaclor e propanil. A determinação destes compostos em matrizes ambientais, de maneira geral, exige o uso de métodos de preparação de amostras seletivos para uma determinada classe de compostos e que envolvem etapas de extração, limpeza e pré-concentração anteriores à determinação cromatográfica ${ }^{6}$.

A extração líquido-líquido é um procedimento de preparo de amostras que consome um volume significativo de solventes orgânicos, longo tempo de extração e intenso trabalho do analista. A técnica de extração em fase sólida, apesar de utilizar pequenas quantidades de solvente para eluir os analitos do sorvente, apresenta problemas de entupimento do sistema de extração e usa grandes volumes de amostra ${ }^{7}$. 
Nos últimos anos, vários avanços na análise de resíduos de pesticidas foram publicados, dentre os quais a microextração em fase sólida (SPME). A aplicação desta técnica de extração foi realizada com sucesso na extração de herbicidas em diferentes tipos de matrizes $^{8-16}$. Desde a primeira citação por Arthur e Pawliszyn ${ }^{17}$, a SPME teve um rápido crescimento em seu uso devido à sua simplicidade e vasto campo de aplicação como alternativa aos métodos tradicionais ${ }^{18}$.

O objetivo deste trabalho foi avaliar, através da técnica de SPME e quantificação por GC-ECD, a presença dos herbicidas alaclor, propanil e atrazina em amostras de água dos rios que abastecem os municípios de Turvo e Meleiro, localizados na região sul de Santa Catarina, os quais são utilizados para o cultivo de arroz irrigado.

\section{PARTE EXPERIMENTAL}

\section{Amostras e reagentes}

As amostras foram coletadas nos municípios Turvo e Meleiro, região sul de Santa Catarina, em junho de 2006. Foram coletadas 5 amostras no rio Amola Faca, 8 amostras no rio Manoel Alves, 1 amostra no rio Itoupava e 1 no rio Jundiá. A coleta foi realizada manualmente empregando frascos de vidro âmbar de $1 \mathrm{~L}$, acondicionados em caixa de isopor e mantidos sobre refrigeração em gelo. As amostras foram transportadas diretamente para o Laboratório Central de Saúde Pública de Santa Catarina, onde foram realizadas as análises. As amostras foram filtradas em membrana de acetato de celulose $(0,45 \mathrm{um})$ e mantidas em freezer a $-18^{\circ} \mathrm{C}$ até o momento da análise.

Os padrões de herbicidas (alaclor, atrazina e propanil) foram adquiridos da Sigma-Aldrich, com grau de pureza superior a 98,3\%. Hidróxido de sódio e cloreto de sódio p.a (99\%) e ácido sulfúrico p.a foram obtidos da Synth. Água milli-Q foi obtida de um sistema de purificação de água Millipore.

As soluções estoques individuais (alaclor, atrazina e propanil) e a mistura dos herbicidas, todas em concentração de $500 \mu \mathrm{g} \mathrm{L}^{-1}$, foram preparadas dissolvendo a quantidade apropriada dos padrões em acetonitrila grau HPLC (MercK). As soluções intermediárias e as de trabalho foram obtidas a partir da diluição da solução estoque por meio de diluições sucessivas. Estas soluções foram utilizadas na determinação da linearidade do método, nas fortificações das amostras e na obtenção das curvas analíticas.

\section{Instrumentação}

As análises foram realizadas em um cromatógrafo a gás Varian CP 3800. A coluna capilar usada foi CP SIL 8CB $50 \mathrm{~m}$ x $0,53 \mathrm{~mm}$, com espessura de filme de 5,0 $\mu \mathrm{m}$ (Varian, EUA). As injeções foram realizadas manualmente no modo splitless com injetor a 280 ${ }^{\circ} \mathrm{C}$. A vazão do gás de arraste nitrogênio $(99,999 \%)$ foi de $1 \mathrm{~mL}$ $\min ^{-1}$ na temperatura inicial do forno da coluna. A programação de temperatura da coluna cromatográfica utilizada para separação dos herbicidas em estudo foi: $60{ }^{\circ} \mathrm{C}$ (4 min) aquecimento até $140{ }^{\circ} \mathrm{C}$ a $30{ }^{\circ} \mathrm{C} \mathrm{min}{ }^{-1}$, de $10{ }^{\circ} \mathrm{C} \min ^{-1}$ até $270{ }^{\circ} \mathrm{C}$ (5 min), e aquecimento para $280{ }^{\circ} \mathrm{C}$ a $50{ }^{\circ} \mathrm{C} \mathrm{min}^{-1}(5 \mathrm{~min})$. A temperatura do detector foi de 300 ${ }^{\circ} \mathrm{C}$.

As identidades dos herbicidas encontrados nas amostras ambientais foram confirmadas através do sistema cromatográfico GC-MS, Saturn 2100 (Varian, EUA) com detector seletivo de massas acoplado e injetor manual split-splitess. A coluna capilar VF$5 \mathrm{~ms}$, com $30 \mathrm{~m}$ x 0,25 mm, com espessura de filme de $0,25 \mu \mathrm{m}$ foi utilizada para a separação dos herbicidas. O gás de arraste hélio foi utilizado com vazão de $1 \mathrm{~mL} \mathrm{~min}{ }^{-1}$ na temperatura inicial do forno da coluna. A programação de temperatura da coluna croma-tográfica foi: $60{ }^{\circ} \mathrm{C}$ (4 min), aquecimento até $140{ }^{\circ} \mathrm{C}$ a $30{ }^{\circ} \mathrm{C} \mathrm{min}$ min $^{-1}$, de $10{ }^{\circ} \mathrm{C}$ $\min ^{-1}$ até $270{ }^{\circ} \mathrm{C}(5 \mathrm{~min})$, e novo aquecimento de $50{ }^{\circ} \mathrm{C} \mathrm{min}^{-1}$ até 280 ${ }^{\circ} \mathrm{C}$ (5 min). As temperaturas do injetor e detector foram de $280 \mathrm{e}$ $300^{\circ} \mathrm{C}$, respectivamente. Os dados foram obtidos usando um software MS Workstation 6.0.

\section{Microextração em fase sólida}

Suporte e fibras de SPME foram adquiridas da Supelco. As extrações dos herbicidas das amostras foram realizadas com fibra de poliacrilato (PA) $85 \mu \mathrm{m}$, sendo usado o modo de extração direto. $\mathrm{O}$ $\mathrm{pH}$ da amostra foi ajustado em 4 e a força iônica em $10 \%$ pela adição de $\mathrm{NaCl}$. A amostra foi mantida sob agitação (700 rpm) e em temperatura de $70{ }^{\circ} \mathrm{C}$ utilizando um agitador magnético modelo VELP Científica com temperatura e agitação ajustáveis. O volume de amostra foi de $15 \mathrm{~mL}$. Os tempos de extração e de dessorção foram de 40 e $5 \mathrm{~min}$, respectivamente.

\section{Validação do método}

Para a determinação dos parâmetros analíticos de mérito, amostras de água destilada foram fortificadas para gerar as seguintes faixas de concentração: 0,066 a 0,$54 ; 0,103$ a 4; 0,065 a $0,1 \mu \mathrm{g} \mathrm{L} \mathrm{L}^{-1}$ para alaclor, atrazina e propanil, respectivamente. O limite de detecção do método ( $\mathrm{LOD}=3,3 \times \mathrm{SD}$ a/b) e o limite de quantificação do método $(\mathrm{LOQ}=10 \times \mathrm{SD}$ a/b), onde: $\mathrm{SD}=$ estimativa do desvio padrão, $\mathrm{a}=$ desvio padrão do coeficiente linear, $b=$ coeficiente angular, estão apresentados na Tabela 1. Os ensaios de precisão e repetibilidade do método foram realizados em amostras de água destilada em 2 níveis de fortificação e seus valores estão na Tabela 2 .

Tabela 1. Parâmetros analíticos obtidos para o método de SPME e GC-ECD

\begin{tabular}{lccccc}
\hline Analito & \multicolumn{5}{c}{ Parâmetros analítico } \\
\hline & $\mathrm{a}$ & $\mathrm{b}$ & $\mathrm{R}$ & $\begin{array}{c}\mathrm{LOD} \\
(\mu \mathrm{g} / \mathrm{L})\end{array}$ & $\begin{array}{c}\text { LOQ } \\
(\mu \mathrm{g} / \mathrm{L})\end{array}$ \\
\hline Alaclor & 1250000 & 2240000 & 0,9957 & 0,049 & 0,147 \\
Atrazina & 10180,12 & 6451,09 & 0,999 & 0,19 & 0,57 \\
Propanil & 60705,32 & 4,474 & 0,9963 & 0,0078 & 0,017 \\
\hline$(\mathrm{n}=5)$ & & & & &
\end{tabular}

$(\mathrm{n}=5)$

Tabela 2. Recuperação dos herbicidas em água destilada para o método proposto

\begin{tabular}{lccccc}
\hline $\begin{array}{l}\text { Recuperação } \\
\text { herbicida }\end{array}$ & \multicolumn{1}{c}{ c teórica } & $\mathrm{c} \exp$ & $\mathrm{DP}^{\mathrm{a}}$ & $\%$ & $\mathrm{DPR}^{\mathrm{b}}$ \\
\hline \multicolumn{7}{c}{$(\mu \mathrm{g} / \mathrm{L})$} & & & \\
\hline Alaclor & $0,147^{\mathrm{c}}$ & 0,17 & 0,03 & 116,9 & 17,3 \\
& 0,27 & 0,20 & 0,04 & 75,4 & 18,1 \\
Atrazina & 0,75 & 0,57 & 0,04 & 76,1 & 6,7 \\
& 3,00 & 3,20 & 0,46 & 106,7 & 14,3 \\
Propanil & 0,017 & 0,015 & 0,003 & 96,2 & 19,2 \\
& 0,032 & 0,035 & 0,007 & 103,9 & 21,2 \\
\hline
\end{tabular}

${ }^{\mathrm{a}}$ Desvio padrão. ${ }^{\mathrm{b}}$ Desvio padrão relativo. ${ }^{\mathrm{c}} \mathrm{n}=3$

\section{RESULTADOS E DISCUSSÃO}

Otimização do procedimento de SPME

\section{Tipo de fibra}

A sensibilidade de um método de SPME depende de uma seleção correta do revestimento e do tipo da fibra, a respeito do com- 
posto de interesse ${ }^{8,19,20}$. Diversos autores têm utilizado as fibras de polidimetilsiloxano (PDMS) e PA para determinação de herbicidas em água ${ }^{21-23}$. Um estudo recente para a determinação de vários herbicidas (incluindo alaclor e atrazina), em amostras de água de rio por SPME e GC-MS, demonstrou que a fibra de PA $(85 \mu \mathrm{m})$ apresenta melhor desempenho que a fibra de PDMS $(100 \mu \mathrm{m})^{11}$. Komatsu e Vaz $^{24}$ otimizaram parâmetros para determinação de multiresíduo de pesticida em água de rio e avaliaram diferentes tipos de fibras (PDMS, carboxen/polidimetilsiloxano (CAR/PDMS), carbowax/divenilbenzeno (CW/DVB) e PA) e concluíram que a fibra com melhor desempenho para atrazina foi a de PA $85 \mu \mathrm{m}$.

Neste estudo as fibras de PDMS $(30 \mu \mathrm{m})$, PA $(85 \mu \mathrm{m}), \mathrm{CAR} /$ PDMS $(75 \mu \mathrm{m})$ e CW/DVB $(65 \mu \mathrm{m})$ tiveram suas eficiências de extração para alaclor, atrazina e propanil comparadas. Conforme é mostrado na Figura 1, a fibra que apresentou melhor desempenho para os herbicidas em estudo foi PA $85 \mu \mathrm{m}$, a qual foi selecionada para os experimentos posteriores.

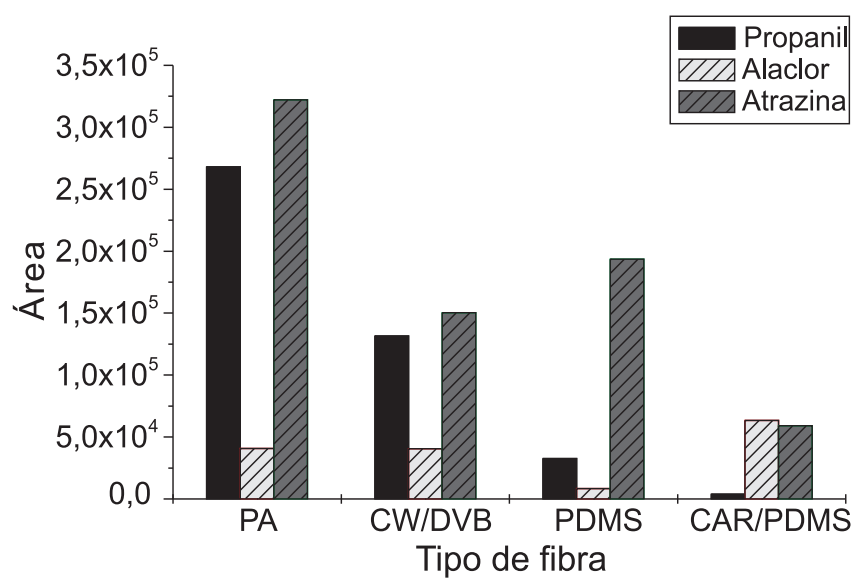

Figura 1. Estudo comparativo da eficiência de extração de diferentes fibras de SPME sobre a extração dos herbicidas propanil, alaclor e atrazina

\section{Modos de extração}

Os modos de extração direto (DI-SPME) e de headspace (HSSPME) foram investigados. O modo HS-SPME não apresentou bons resultados para dois dos herbicidas estudados (alaclor e propanil), provavelmente devido à alta polaridade e baixa volatilidade destes compostos. Desta maneira, o modo de extração direto foi selecionado. Cabe enfatizar que a eficiência de cada modo depende das propriedades dos analitos e da matriz. Em geral, o DI-SPME é mais sensível que a HSSPME para analítos presentes em amostras líquidas. No entanto, compostos não voláteis presentes na amostra ficam retidos na fibra, podendo reduzir a sua vida útil e a repetibilidade de extração ${ }^{25}$.

\section{Influência do $\mathrm{pH}$}

Segundo Gonçalves e Alpendurada ${ }^{10}$, a afinidade dos analítos para o adsorvente age primeiramente por interações hidrofóbicas podendo ser ativada mantendo as moléculas na forma não ionizadas. Desde modo, a influência do pH (3, 4, 5, 7 e 8) sobre a eficiência de extração dos herbicidas alvos deste estudo foi verificada. A Figura 2 demonstra que para os herbicidas estudados, o mais afetado pela variação do $\mathrm{pH}$ foi o alaclor, que teve sua extração diminuída em pH mais básico. Para os demais compostos não houve variação significativa da eficiência de extração para os diferentes valores de $\mathrm{pH}$. As extrações realizadas em pH ácido (3 e 4) apresentaram os melhores resultados, sendo o $\mathrm{pH} 4$ selecionado, o qual pode levar à redução de possíveis danos à fibra, o que resultaria na diminuição do tempo de vida útil da mesma.

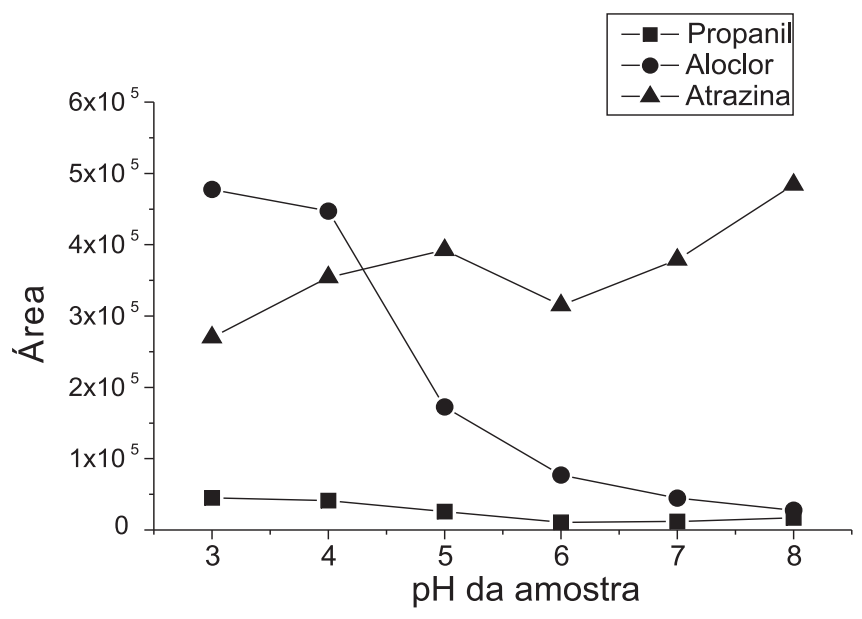

Figura 2. Influência do pH sobre a extração dos herbicidas propanil, alaclor e atrazina utilizando fibra de PA $85 \mathrm{~mm}$

Influência da adição de sal

A influência da adição de cloreto de sódio em concentrações de 4; 10 e 23,3\% sobre a eficiência de extração dos herbicidas foi investigada. A atrazina foi o herbicida que apresentou um efeito significativo na eficiência de extração com a adição de $\mathrm{NaCl}$ em relação aos outros herbicidas. No entanto foi feita a opção pela concentração salina de $10 \%$ devido às dificuldades encontradas em se trabalhar com concentrações mais elevadas de sal, principalmente pela saturação da fibra de PA $85 \mu \mathrm{m}$.

\section{Velocidade de agitação da amostra}

De acordo com alguns trabalhos ${ }^{10,24}$, a velocidade de agitação é utilizada para facilitar o transporte de massa entre a fase aquosa e a fibra.

Assim, a influência da velocidade de agitação da amostra entre 0 (sem agitação) e $1000 \mathrm{rpm}$ foi avaliada. Este estudo demonstrou que a eficiência da extração foi melhor em velocidades de agitação de $1000 \mathrm{rpm}$, exceto para o propanil onde não foi observada praticamente nenhuma alteração da eficiência da extração em função da velocidade de agitação. No entanto, devido a condições laboratoriais fez-se opção pela velocidade de $700 \mathrm{rpm}$.

\section{Temperatura da amostra}

Segundo Gonçalves e Alpendurada ${ }^{10}$, variações de temperatura afetam a cinética e a termodinâmica do processo de extração. Temperaturas acima de $60{ }^{\circ} \mathrm{C}$ podem ser prejudiciais para extração dos pesticidas clorados. A maioria dos autores concorda com a importância da temperatura de extração por SPME para pesticida e recomenda empregar temperaturas até $100{ }^{\circ} \mathrm{C}$, dependendo do pesticida em estudo e do tipo de matriz ${ }^{20,22,26}$.

Assim, o efeito da temperatura da amostra $\left(27,40,70\right.$ e $\left.80{ }^{\circ} \mathrm{C}\right)$ sobre o rendimento de extração foi estudado. Na Figura 3 fica evidenciado que com a elevação da temperatura até $70{ }^{\circ} \mathrm{C}$ houve uma melhora na extração simultânea dos herbicidas. Temperaturas acima de $70{ }^{\circ} \mathrm{C}$ diminuíram o rendimento de extração dos herbicidas.

\section{Volume da amostra}

O volume da amostra é um parâmetro importante na otimização por SPME porque está relacionado diretamente com a sensibilidade do método ${ }^{27}$. Neste estudo foram otimizados quatro volumes da amostra (5; 10; 15 e $45 \mathrm{~mL}$ ). Os volumes de 10 e $15 \mathrm{~mL}$ apresentaram os melhores resultados. Desta maneira, fez-se a escolha pelo volume de $15 \mathrm{~mL}$. Geralmente, a quantidade de analito absorvida na fase estacio- 


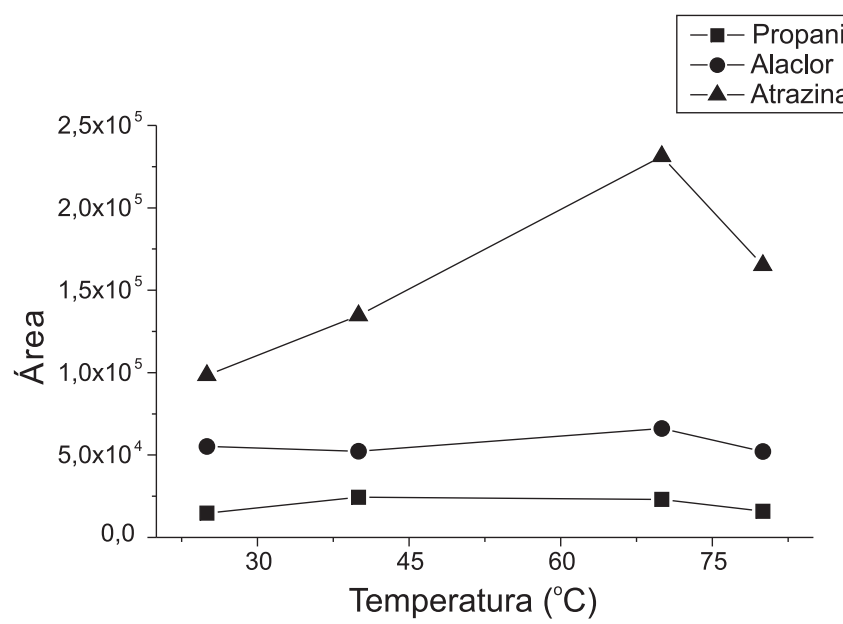

Figura 3. Influência da temperatura da amostra sobre a extração dos herbicidas propanil, alaclor e atrazina

nária aumenta quando o volume da amostra aumenta e, conseqüentemente, a sensibilidade também aumenta ${ }^{28}$. Poucos estudos relatam otimização de volume da amostra por SPME. Em alguns dos poucos estudos onde o volume de amostra foi otimizado, os volumes de amostra melhores encontrados ficaram entre 4 e $120 \mathrm{~mL}^{9}$.

\section{Tempo de extração}

Após a otimização do tipo de fibra, modo de extração, $\mathrm{pH}$ da amostra, concentração salina, velocidade de agitação, volume e temperatura, foi estudado o tempo de extração no intervalo de 20 a 130 min. O resultado deste estudo indicou que mesmo após 130 min o equilíbrio do sistema não foi alcançado. Devido ao fato que o equilíbrio não é necessário desde que as condições de extração sejam mantidas, e considerando a possibilidade da implantação do método em análise de rotina, foi feita a opção pelo tempo de extração de $30 \mathrm{~min}$.

\section{Efeito de memória}

O método baseado na SPME pode estar sujeito a efeitos de memória. Estes efeitos são caracterizados pela dessorção incompleta dos analitos adsorvidos na fibra, podendo ser mais acentuada em compostos com maior afinidade pelo recobrimento da fibra ${ }^{27}$. Para verificar o efeito de memória (efeito "carryover") foram testados 3 diferentes tempos: 3, 5 e 10 min de dessorção na temperatura de $280{ }^{\circ} \mathrm{C}$. Com 3 min de dessorção os resíduos apresentados já foram menores que 5\%; no entanto, para garantir a completa dessorção foi padronizado o tempo de $5 \mathrm{~min}$.

\section{Amostras ambientais}

O método descrito foi aplicado para determinação dos herbicidas em amostras de água de rio, que foram analisadas por GC-ECD e confirmadas no GC-MS. As amostras foram coletadas de mananciais que são a principal fonte de abastecimento de água potável dos municípios de Turvo e Meleiro, na região sul do estado. Nestes locais o cultivo de arroz irrigado é uma das mais importantes atividades agrícolas.

Quanto aos resultados encontrados, foram detectados herbicidas em todas as amostras analisadas e com níveis de concentrações estimadas variando de 0,14 a $0,72 \mu \mathrm{g} \mathrm{L}^{-1}$ para alaclor e 0,02 a 0,05 $\mu \mathrm{g} \mathrm{L}^{-1}$ para propanil. Cabe enfatizar que foi detectada a presença de pelo menos um dos herbicidas nos rios estudados. Quanto ao rio Manoel Alves, onde foi coletado um número maior de amostras (8), em 4 delas foi detectado alaclor e em 2 a presença de propanil com níveis de 0,14 a $0,32 \mu \mathrm{g} \mathrm{L}^{-1}$, respectivamente. No rio Amola Faca foram analisadas 5 amostras, sendo que 2 apresentaram alaclor com níveis de 0,31 a $0,45 \mu \mathrm{g} \mathrm{L}^{-1}$ e uma 1 apresentou propanil em concentração de $0,02 \mu \mathrm{g} \mathrm{L}^{-1}$. No rio Itoupava (1 amostra coletada) foi detectada a presença de propanil ao nível de $0,03 \mu \mathrm{g} \mathrm{L}^{-1}$. No rio Jundiá constatou-se a presença de alaclor com níveis de $0,72 \mu \mathrm{g} \mathrm{L}$ ${ }^{1}$ e propanil em $0,05 \mu \mathrm{g} \mathrm{L}^{-1}$ na única amostra coletada. Em estudos realizados por Carabias-Martinez et al. ${ }^{11}$, para determinação de herbicidas em água de rio usando a técnica de SPME, foi detectada a presença de alaclor nas amostras analisadas. Estes estudos também mostraram que não foi detectada a presença de atrazina.

Devido às dificuldades de correlacionar nossos dados com literatura brasileira para a mistura destes herbicidas em água, relacionamos com alguns trabalhos científicos internacionais. Lambropoulou et al. ${ }^{29}$, em águas de Rio Kalamas, detectaram atrazina e alaclor em concentrações médias que variaram de 0,05 a $0,008 \mu \mathrm{g}$ $\mathrm{L}^{-1}$, respectivamente. Outros autores ${ }^{11}$ também analisaram águas de rios e subterrâneas da Espanha e detectaram a presença de atrazina com níveis de 0,74 a $0,99 \mu \mathrm{g} \mathrm{L}^{-1}$. Lambropoulou et al..$^{12}$ encontraram atrazina em amostras de água do rio Kalamas e os níveis variaram de 0,002 a 0,23 $\mu \mathrm{g} \mathrm{L}^{-1}$. Em 2006, Konstantinou et al. ${ }^{15}$ publicaram uma revisão avaliando os níveis de contaminação por pesticidas das águas dos rios de 1993 a 2004, sendo que das várias amostras analisadas, $23 \%$ apresentaram contaminação por atrazina e os níveis variaram de 0,002 a $0,6 \mu \mathrm{g} \mathrm{L}^{-1}$.

Neste estudo, metade das amostras analisadas apresentou resíduos de herbicidas em níveis abaixo do permitido pela legislação brasileira $\left(2 \mu \mathrm{g} \mathrm{L} \mathrm{L}^{-1}\right.$ para atrazina e $20 \mu \mathrm{g} \mathrm{L}^{-1}$ para alaclor e propanil). No entanto, cabe ressaltar que foram detectadas as presenças de alaclor em nossas amostras acima dos níveis aceitáveis pela legislação européia (máximo de $0,1 \mu \mathrm{g} \mathrm{L} \mathrm{L}^{-1}$ para pesticidas individuais e $0,5 \mu \mathrm{g} \mathrm{L}^{-1}$ para pesticidas totais). De acordo com dados da literatura, herbicidas como atrazina têm sido freqüentemente detectados em água naturais ${ }^{30}$; embora ainda estejam sendo usados em muitos países, foram recentemente banidos o seu uso na Itália e na Alemanha, devido ao seu potencial tóxico.

\section{CONCLUSÃO}

Todas as amostras de água analisadas apresentaram contaminação por pelo menos um dos herbicidas, e os níveis variaram de 0,14 a $0,72 \mu \mathrm{g} \mathrm{L}^{-1}$ para alaclor e 0,02 a $0,05 \mu \mathrm{g} \mathrm{L} \mathrm{L}^{-1}$ para propanil. Mesmo que não tenha sido detectada em nosso estudo uma concentração dos herbicidas alaclor, propanil e atrazina acima da permitida pela Legislação Brasileira, cabe enfatizar a importância de monitorar estes herbicidas, principalmente atrazina. A EPA estima que a exposição de $3 \mu \mathrm{g} \mathrm{L}^{-1}$ de atrazina, que é o limite máximo estabelecido, apresenta risco de 1/100.000 da pessoa ter câncer durante a vida. Daí a necessidade de métodos simples, rápidos e práticos, como a SPME, para serem aplicadas em análise de rotina. A técnica de SPME-GC-ECD apresentou bons resultados analíticos quanto à linearidade, limite de detecção e determinação, além de reduzir o tempo de análise, ser livre de solvente e atender à Legislação Brasileira e à Comunidade Européia.

\section{REFERÊNCIAS}

1. Hadlich, G. M.; Dissertação de Mestrado, Universidade Federal de Santa Catarina, Brasil, 1997

2. Campanhola, C.; Rodrigues, G. S.; Bettiol, W.; Seminário Internacional de racionalização do uso de pesticidas no cone sul, Campinas, Brasil, 1997.

3. Paschoal, A. D.; Pragas, praguicidas e a crise ambiental: problemas e soluções, Fundação Getúlio Vargas: Rio de Janeiro, 1979.

4. European Commission Regulation; Drinking Water Guideline 80/779/EEC, Official Journal of the European Communities, 1980, L 229. 
5. Ministério da Saúde. Diário Oficial da União, Brasília, DF: MS; 2004 Portaria No. 380 518/2004.

6. Lacorte, S.; Guiffard, I.; Fraisse, D.; Barceló, D.; Anal. Chem. 2000, 72, 7.

7. Huang, Y.; Yang, Y-C.; Shu, Y. Y.; J. Chromatogr., A 2007, 1140, 35; Lino, C. M.; Silveira, M. I. N.; J. Chromatogr., A 1997, 769, 275.

8. Cancho, B.; Ventura, F.; Galceram, M. T.; J. Chromatogr., A 2001, 943, 1.

9. Gonzálvez-Barreiro, C.; Lores, M.; Casais, M. C.; Cela, R.; J. Chromatogr., A 2000, 896, 373.

10. Gonçalves, C.; Alpendurada, M. F.; J. Chromatogr., A 2002, 968, 177.

11. Carabias-Martínez, R.; Garcia-Hermida, C.; Rodriguez-Gonzalo, E.; SorianoBravo, F. E.; Hernández-Méndez, J.; J. Chromatogr., A 2003, 1002, 1.

12. Lambropoulou, D. A.; Konstantinou, I. K.; Albanis, T. A.; J. Chromatogr., A 2000, 893, 143.

13. López-Blanco, M. C.; Blanco-Cid, S.; Cancho-Grande, B.; Simal-Gándara; J. Chromatogr., A 2003, 984, 245.

14. Bangheri, H.; Khalilian, F.; Anal. Chim. Acta 2005, 537, 81.

15. Konstantinou, I. K.; Hela, D. G.; Albanis, T. A.; Environ. Pollut. 2006, 141, 555.

16. Zhao, E-C.; Shan, W-L.; Jiang, S-R.; Liu, Y.; Zhou, Z-Q.; Microchem. J. 2006, 83, 105 .
17. Arthur, C. L.; Pawliszyn, J.; Anal. Chem. 1990, 62, 2145.

18. Augusto, F.; Valente, A. L. P.; Quim. Nova 2000, 23, 523.

19. Alpendurada, M. F.; J. Chromatogr., A 2000, 889, 3.

20. Lord, H.; Pawliszyn, J.; J. Chromatogr., A 2000, 885, 153.

21. Lambropoulou, D. A.; Albanis. T.; J. Chromatogr. A 2001, 922, 243.

22. Beltran. J.; López, F. J.; Hernández, F.; J. Chromatogr. A 2000, 885, 389.

23. Dugay, J.; Miége, C.; Hennion, M. C.; J. Chromatogr., A 1998, 795, 27.

24. Komatsu, E.; Vaz, J. M.; Quim. Nova 2004, 27, 720.

25. Katoaka, H.; Lord, H. L.; Pawliszyn, J.; J. Chromatogr., A 2000, 880, 35

26. Correia. M.; Delerue-Mato, S. C.; Alves A.; J. Chromatogr., A 2000, 889, 59.

27. Pawliszyn, J.; Solid Phase Microextraction: Theory and Practice, $1^{\text {st }}$ ed., Wiley-VCH: New York, 1997.

28. Krutz, L. J.; Senseman, S. A.; Sciumbato, A. S.; J. Chromatogr., A 2003, 999, 103.

29. Lambropoulou, D. A.; Sakkas, V. A.; Hela, G. D.; Albanis, T. A.; J. Chromatogr., A 2002, 963, 107.

30. WHO; Drinking Water Quality: Guidelines for selected herbicides, Environmental Health Criteria, 1987, p. 27. 